\title{
Gastroesophageal Reflux Disease and Need for Antireflux Surgery in Children with Cystic Fibrosis: A Systematic Review on Incidence, Surgical Complications, and Postoperative Outcomes
}

\author{
Jessica Ng1® Florian Friedmacher $^{1,2}$ Caroline Pao ${ }^{1}$ Paul Charlesworth ${ }^{1}$ \\ ${ }^{1}$ Department of Paediatric Surgery, Royal London Hospital, London, \\ United Kingdom \\ 2 Department of Pediatric Surgery, University Hospital Frankfurt, \\ Goethe University Frankfurt, Frankfurt (Main), Germany \\ Address for correspondence Florian Friedmacher, MD, MSc (RCSI), \\ PhD, Department of Pediatric Surgery, University Hospital Frankfurt, \\ Goethe University Frankfurt, Theodor-Stern-Kai 7, 60590 \\ Frankfurt/Main, Germany (e-mail: florian.friedmacher@nhs.net).
}

Eur J Pediatr Surg 2021;31:106-114.

\begin{abstract}
Keywords

- gastroesophageal reflux

- cystic fibrosis

- fundoplication

- antireflux surgery

- pediatric

Introduction Gastroesophageal reflux disease (GERD) is associated with accelerated decline in lung health in children with cystic fibrosis (CF). Thus, antireflux surgery (ARS) is offered to a selected CF cohort with refractory GERD, but outcomes remain poorly investigated. This study aimed to determine the incidence of GERD in children with CF and to evaluate complications and outcomes of ARS.

Materials and Methods A systematic literature-based search was conducted using various online databases according to Preferred Reporting Items for Systematic Reviews and Meta-Analyses guidelines. The number of GERD cases in pediatric CF cohorts who underwent diagnostic investigation(s) was recorded. Data on postoperative complications and outcomes (including symptoms, lung function, and nutritional status) following ARS were analyzed.

Results Ten articles ( $n=289$ patients) met the defined inclusion criteria ( $51 \%$ male; age range, 0.5 month-36 years). The overall incidence of GERD was $46 \%$ (range, 19 $81 \%)$, derived from seven studies ( $n=212$ patients). Four publications $(n=82$ patients) reported on ARS due to uncontrolled GERD. All ARSs were Nissen fundoplication (majority with gastrostomy placement). Major postoperative complications occurred in 15 (18\%) patients, two required redo-ARS. Median follow-up time was 2 years (range, 3 months- 6 years); 59\% showed symptom improvement, and pulmonary exacerbations and decline in lung function were reduced. Nutritional status mainly improved in milder CF cases. There were no deaths related to ARS.

Conclusion Approximately half of pediatric CF patients have GERD. Published data for children with CF are limited and heterogeneous in terms of GERD diagnosis and outcomes following ARS. However, ARS has shown to slow the deterioration of lung function in CF.
\end{abstract}

received

May 15, 2020

accepted after revision

September 10, 2020

published online

November 17, 2020
DOI https://doi.org/ 10.1055/s-0040-1718750. ISSN 0939-7248.

\footnotetext{
(C) 2020. The Author(s).

This is an open access article published by Thieme under the terms of the Creative Commons Attribution-NonDerivative-NonCommercial-License, permitting copying and reproduction so long as the original work is given appropriate credit. Contents may not be used for commercial purposes, or adapted, remixed, transformed or built upon. (https://creativecommons.org/ licenses/by-nc-nd/4.0/) Georg Thieme Verlag KG, Rüdigerstraße 14, 70469 Stuttgart, Germany
} 


\section{Introduction}

Gastroesophageal reflux allows the passage of gastric contents into the esophagus with or without regurgitation or vomiting. ${ }^{1}$ In children with cystic fibrosis (CF), gastroesophageal reflux disease (GERD) is associated with accelerated decline in lung health with increased pulmonary exacerbations and failure to thrive. ${ }^{2-4}$ GERD is more common in CF than in normal patients as a result of altered gastric emptying (GE), decreased lower esophageal sphincter (LES) pressure, increased frequency of transient LES relaxation, poorer esophageal peristalsis, and high fat due to pancreatic insufficiency. ${ }^{5}$ Investigations for GERD include 24-hour pH monitoring, combined pH-impedance study, upper gastrointestinal (UGI) contrast, GE scintigraphy, and esophagogastroduodenoscopy (EGD) with biopsy. Antireflux medications are commonly used to manage GERDrelated symptoms in CF patients and to control progression of disease.

In children, antireflux surgery (ARS) is generally reserved for cases with failed optimal medical therapy for GERD. Fundoplication in the pediatric age group has an overall success rate of $86 \%$ in terms of complete relief of GERD symptoms. ${ }^{6}$ However, adverse outcomes, including recurrence and mortality, are frequently reported in children with severe comorbidities such as neurological impairment compared with normal cases. ${ }^{7}$ Although ARS is an established treatment option for children with $\mathrm{CF}$, postoperative success and complication rates remain poorly documented.

The aim of this study was to determine the incidence of GERD in pediatric cohorts with CF, and to evaluate surgical complications and postoperative outcomes of ARS based on a systematic review of the published literature.

\section{Materials and Methods}

\section{Information Sources and Literature-Based Search}

To identify as many scientific articles as possible of reported GERD cases in pediatric CF cohorts, a systematic literaturebased search was performed in accordance with the Preferred Reporting Items for Systematic Reviews and Meta-Analyses guidelines using PubMed, EMBASE, Scopus, Web of Science, CINAHL, Centre for Reviews and Dissemination, and Cochrane Central Register of Controlled Trials. ${ }^{8}$ These electronic databases were last accessed on May 15, 2020, using a combination of the following linked Medical Subject Headings and search terms: "cystic* fibrosis" OR "mucoviscidosis" AND "gastro? esoph* reflux" OR "gastric* acid* reflux*." The search was restricted to articles published in English. In addition, reference lists of identified publications were manually searched for further studies and duplicate listed items were removed.

\section{Selection Criteria and Data Extraction}

All identified articles were reviewed by title, keywords, and abstract by one of the authors (F.F.). Publications that did not contain original research data (e.g., editorial commentaries, letters, or reviews) and experimental animal studies were excluded. Additional exclusion criteria were case reports and CF not being the primary pathology. Only articles reporting on pediatric CF cohorts in which GERD was investigated with established diagnostic tests (i.e., UGI contrast study, $\mathrm{pH}$ or pH-impedance monitoring, GE scintigraphy, EGD, and esophageal biopsy) were considered as relevant. An unblinded, systematic full-text assessment of selected publications was independently performed by two of the authors (J.N. and F.F.) and all studies not giving accurate information regarding the incidence of GERD in the respective pediatric cohorts with $\mathrm{CF}$ were excluded. In cases of double-published or overlapping datasets from the same institution, more recent articles or those with the larger cohort size were considered for analysis. Any discrepancies between the authors were resolved by mutual consensus.

Data from selected publications were extracted into an electronic datasheet in a standardized manner, including study characteristics (i.e., authors, publication year, geographical region, study design, and cohort size) and patient characteristics (i.e., age, gender, GERD investigation[s], treatment modality, follow-up time, and outcome measures). Primary outcome measures were severity of GERD symptoms (including those related to the gastrointestinal and airway system), pulmonary exacerbations, lung function, weight, and nutritional status. Secondary outcome measures were postoperative complications and need for redo ARS. As not all of the included articles reported on surgical complications and outcomes after ARS, each specific complication and outcome parameter were recorded as the number of patients in which that item was present divided by the total number of patients in the cohorts that presented data on that item. This enabled the calculation of incidence rates for each specific complication and outcome parameter.

\section{Evidence Ranking and Quality Assessment}

The evidence level of the included publications was ranked according to the Oxford Centre for Evidence-Based Medicine (OCEBM) classification system. ${ }^{9}$ This is a hierarchical grading system, which classifies studies into Levels 1 to 5 , with Level 1 representing the highest level of evidence consisting of systematic reviews of randomized controlled trials (RCTs). To reach Level 2, at least one well-designed RCT is required, whereas nonrandomized controlled cohort or follow-up studies are allocated to Level 3. Level 4 is characterized by case-control or poor-quality cohort studies, and Level 5 is assigned to mechanism-based reasoning.

The quality of selected articles was evaluated using the methodological index for nonrandomized studies (MINORS). ${ }^{10}$ This validated instrument contains eight methodological items for assessment of noncomparative studies, each being scored as 0 (not reported), 1 (reported but inadequate), or 2 (reported and adequate). Thus, the ideal global score being 16 .

\section{Results}

\section{Literature-Based Search}

The initial search yielded a total of 710 publications, of which 708 were identified by electronic database searching and 2 from cross-referencing ( - Fig. 1). After the removal of 456 duplicate listed items, 254 titles, keywords, and abstracts 


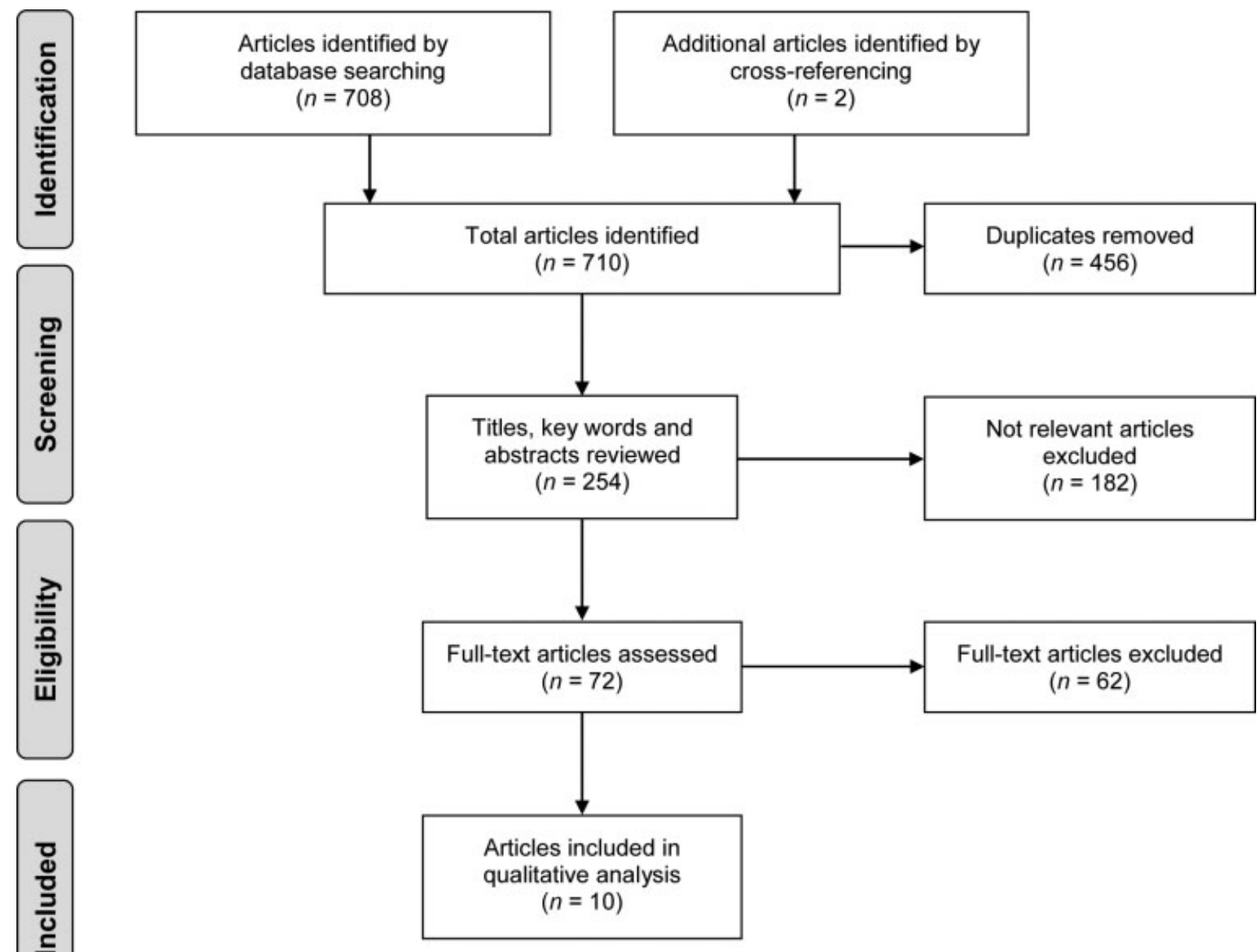

Fig. 1 Flowchart illustrating the different phases of the literature-based search and selection process.

were screened. Of these, 182 were considered as not relevant and excluded. The remaining 72 articles were assessed in full text for eligibility and 62 were excluded because they did not address all of the selection criteria. In total, data from 10 studies (published between 1982 and 2016) reporting on 289 pediatric CF patients met defined inclusion criteria and were included in the qualitative analysis.

\section{Overall Incidence of GERD}

Seven of the 10 articles were eligible for detailed analysis of GERD incidence in pediatric CF cohorts (- Table 1). ${ }^{11-17}$ In total, there were 212 children with CF available for analysis, of which 108 (51\%) were male. The median number of patients in the study cohort was 28 (range, 8-44) with an age range from 0.5 months to 20 years. Primary investigation modality for GERD and diagnostic criteria were heterogeneous, including UGI contrast study, $\mathrm{pH}$, or $\mathrm{pH}$-impedance monitoring. The criteria used for diagnosing GERD for $\mathrm{pH}$ studies differed with reflux index (RI) thresholds ranging from $>1$ to $>10 \%$. Diagnostic criteria for $\mathrm{pH}$-impedance monitoring were RI $>5$ and $>6 \%$. Overall, $46 \%$ (97/212) of identified pediatric CF cases were diagnosed with GERD. The median reported GERD incidence was 50\% (range, 20-81\%).

\section{ARS-Related Complications and Postoperative Outcomes}

Four of the 10 studies were eligible for detailed analysis of ARS-related outcomes and complications (-Table 2). ${ }^{11,18-20}$ A total of 82 infants and children with CF underwent ARS. The median number of CF cases in these articles was 15 (range, 448). Patients were aged 1 month to 36 years, of which 42 (51\%) were male.

Various investigation modalities were utilized to define and assess GERD including $\mathrm{pH}$ monitoring $(n=68$ [83\%]; positive result in 97\%), UGI contrast study $(n=29$ [35\%]; positive result in $48 \%$ ), GE scintigraphy ( $n=10$ [12\%]; positive result in 70\%), and EGD with or without biopsy ( $n=9$ [11\%]; positive result in $100 \%$ ).

All ARSs were either laparoscopic or open Nissen fundoplication. The majority of patients underwent gastrostomy placement either at the same time or before ARS. The median follow-up time was 2 years (range, 3 months- 6 years).

Primary outcome measures were heterogeneous across all publications and included GERD symptoms, pulmonary exacerbation episodes, rate of change in lung function (i.e., forced expiratory volume in 1 second $\left.\left[\mathrm{FEV}_{1}\right]\right)$, nutritional measurements (i.e., weight and/or body mass index [BMI]). Three 


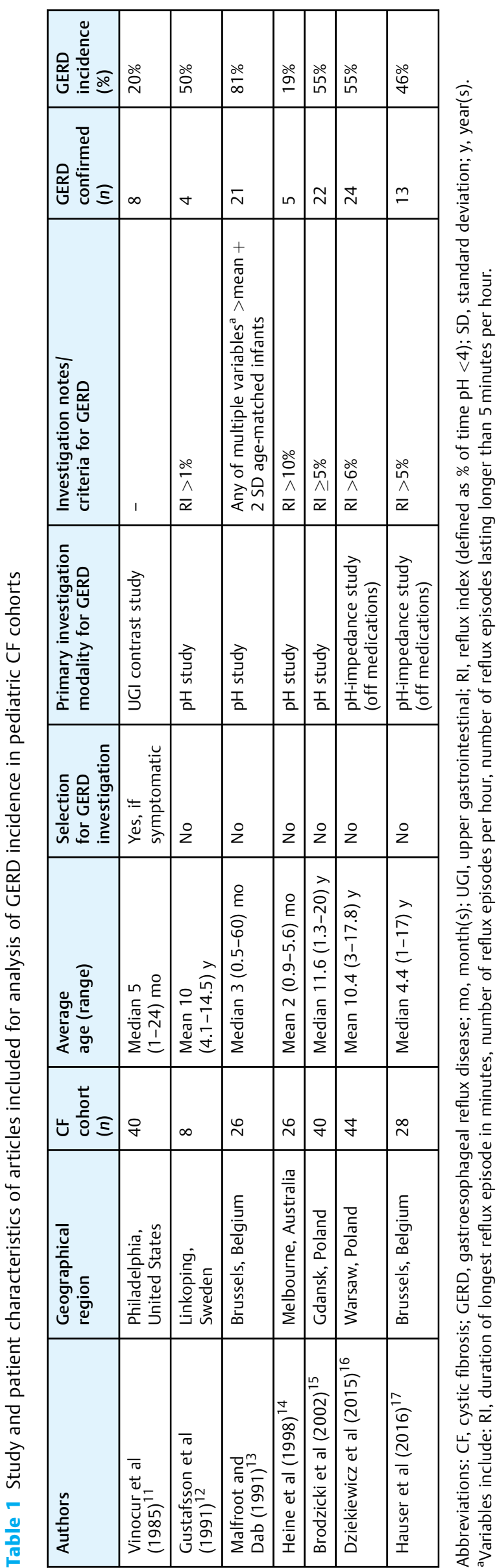

studies, with a total of 34 pediatric CF patients with GERD, reported gastrointestinal symptoms following ARS. ${ }^{11,18,19}$ Of these, 20 (59\%) showed symptom improvement, whereas 14 (41\%) had persistent or worsening of symptoms. Sheikh et al analyzed in their series 48 children with CF after fundoplication and demonstrated an average reduction of 1.3 episodes per year in pulmonary exacerbations requiring intravenous antibiotics. $^{20}$ They also demonstrated a significantly slowed decline in $\mathrm{FEV}_{1}$ over 2 years; the mean $\% \mathrm{FEV}_{1} 2$ years before ARS was $79 \%$ with a mean change by $-13 \%$, compared with a mean change of +1.52 years post-ARS. The nutritional status was assessed in three articles consisting of 78 pediatric $C F$ cases that underwent fundoplication. ${ }^{11,19,20}$ Overall, there was weight gain but no significant improvement in BMI. Vinocur et al's cohort of infants had gastrostomy inserted concurrently with ARS and all showed significantly increased weight and weight centiles. ${ }^{11}$ The majority of Boesch and Acton's series of older children had gastrostomy concurrently with their fundoplication; however, they only found a significant increase in BMI in cases with mild lung disease (i.e., $\mathrm{FEV}_{1} \geq 60 \%$ ). ${ }^{19}$ Sheikh et al's patients had a significant weight gain but no improvement in BMI or BMI\%. ${ }^{20}$ Two-thirds of their children had gastrostomy inserted either concurrently or before ARS. It was apparent that the groups with gastrostomy supplemental feeding and those with mild lung disease demonstrated a significant weight gain. ${ }^{20}$

Major postoperative complications following fundoplication were reported in 15 (18\%) patients, including loose wrap ( $n=6 ; 7 \%)$, paraesophageal hernia $(n=5 ; 6 \%)$, perforation $(n=1 ; 1 \%)$, wrap disruption $(n=1 ; 1 \%)$, esophageal stricture $(n=1 ; 1 \%)$, and splenic infarction $(n=1 ; 1 \%)$. Two $(2 \%)$ patients eventually required redo ARS. There were no deaths as a result of ARS.

\section{Evidence Ranking and Quality Assessment}

The level of evidence of included publications was categorized in each case as OCEBM Level 4. Six were retrospective observational studies, whereas in four, the data were collected prospectively. According to the MINORS instrument, the methodological quality of these articles ranged between 7 and 12 (-Table 3), and therefore, none of them reached the ideal global score of 16 . None of the articles provided an unbiased assessment of the individual study end points or prospective calculation of the sample size.

\section{Discussion}

GERD is prevalent in pediatric patients with $\mathrm{CF}$, which may manifest in early infancy and often persists throughout childhood with deleterious effects on lung function and growth. Our systematic review revealed that $46 \%$ of children with CF have GERD. This is in comparison to a reported prevalence of $6.2 \%$ in non-CF children in a large cross-sectional observational study on more than 10,000 subjects. $^{22}$ Hence, all CF patients with clinical signs of GERD should be aggressively managed on an escalating pathway of medical and surgical therapies. Initially, antireflux medications control symptoms while limiting the adverse effects of GERD. The majority of medically managed 


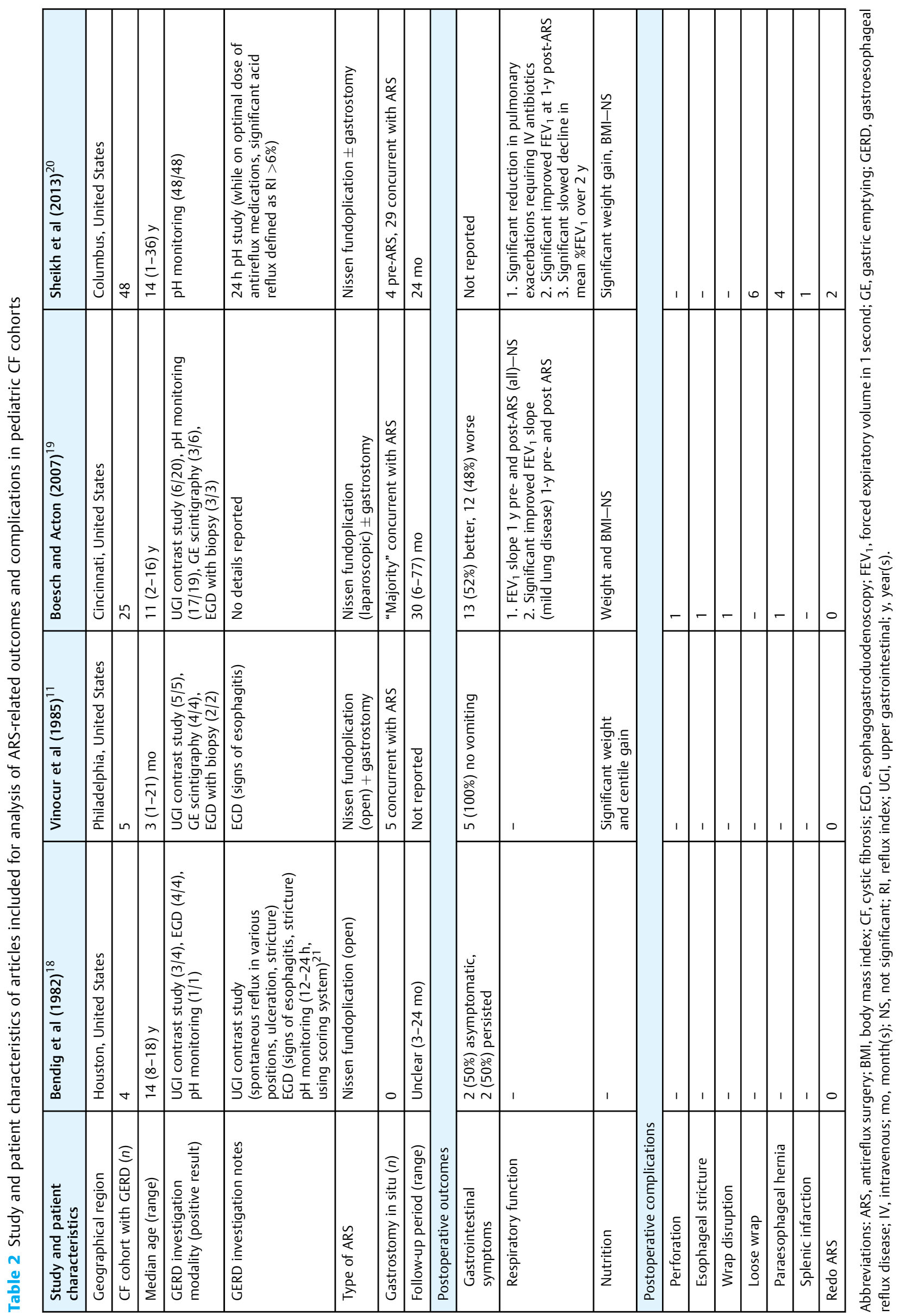




$$
[
$$


GERD is successful, but $10 \%$ of cases fail optimal therapy with persisting GERD necessitating ARS. ${ }^{20}$

\section{GERD Diagnosis and Incidence in CF Children}

The latest joint recommendations of the North American and European Societies for Pediatric Gastroenterology, Hepatology and Nutrition (NASPGHAN and ESPGHAN) were published in 2018. ${ }^{1}$ Both societies currently recommend that routine diagnosis for GERD is clinical in infants. In older children with typical gastrointestinal symptoms, diagnosis may be based on trial of proton pump inhibitors for 4 to 8 weeks. The use of $\mathrm{pH}$ or pH-impedance study, the latter being preferable, is recommended to assess efficacy of GERD treatment in high-risk patients such as those with CF. This "gold standard" was not employed in the majority of identified publications presumably due to their invasive nature. The investigations used to diagnose GERD were heterogeneous among the analyzed articles, including UGI contrast study, $\mathrm{pH}$ or $\mathrm{pH}$-impedance monitoring, GE scintigraphy, and EGD with or without biopsy.

There are no universal "normal values" in the pediatric population, so an additional limiting factor is that studies used different criteria to diagnose GERD when using $\mathrm{pH}$ and pH-impedance monitoring. In three articles published between 1991 and 2002, GERD was diagnosed using pH study with varying RIs: $>1,>5,>10 \%$, or used age-matched means and standard deviations. ${ }^{12-15}$ Two later articles (published in 2015 and 2016) used pH-impedance monitoring to investigate GERD and the diagnosis were based on RIs $>6$ and $>5 \%$, respectively. ${ }^{16,17}$ However, symptom correlation for $\mathrm{pH}$ or $\mathrm{pH}$-impedance studies was not included in their methodologies. Dziekiewicz et al specifically reported that they were unable to calculate symptom correlation parameters as patients or their parents did not note any specific symptoms. ${ }^{16}$ This is not surprising as children often have poor recognition of their chronic symptoms.

In addition to the above-cited GERD investigations, children with CF often undergo computed tomography to assess for lung damage and bronchiectasis. The findings of early or deteriorating features may prompt further GERD assessment. NASPGHAN and ESPGHAN continue to recommend EGD with biopsies in patients with suspected complications of GERD prior to escalation of therapy. ${ }^{1}$ Delayed GE is implicated in the pathogenesis of GERD in CF children. Although GE scintigraphy is not recommend to routinely diagnose GERD, it may be useful in children with CF when escalating to prokinetic therapies. ${ }^{1}$ UGI contrast study is not recommended for routine diagnosis of GERD. ${ }^{1}$ Its predominant use is to investigate whether other anatomical anomalies exist that may exacerbate symptoms of GERD or cause similar symptoms to gastroesophageal reflux which is useful prior to ARS.

\section{ARS-Related Outcomes and Complications}

The aim of ARS in children with CF and GERD refractory to medical management is preservation of lung function to extend life expectancy and to improve their quality of life. Currently, a paucity of literature data exists on ARS in this cohort. The limited data have nonunified and subjective outcome measures evaluating gastrointestinal symptoms, $\mathrm{CF}$ exacerbation, lung function $\left(\mathrm{FEV}_{1}\right)$, weight, and BMI.

\section{Gastrointestinal Symptoms}

Just more than half of the identified children with CF had symptomatic improvement in gastrointestinal symptomatology following ARS, while the remaining half had worsening or persistence of symptoms. Using symptom relief as an indicator of success of ARS in pediatric CF cases may indicate improved quality of life. However, many children with CF may have silent reflux; moreover, gastrointestinal symptom relief may not correlate with respiratory symptoms and associated lung function.

\section{Lung Function}

The most important question the surgeon needs to answer is that by doing ARS, are we preserving pulmonary function in this cohort with refractory GERD? Data on lung function was presented in two larger studies, generally showing improved lung function. ${ }^{19,20}$ Sheikh et al also demonstrated a reduction in the number of pulmonary exacerbation episodes requiring intravenous antibiotics and improved $\mathrm{FEV}_{1}$ slopes 1 year after fundoplication. ${ }^{20}$ Interestingly, progression of lung disease was slowed in both those with severe and mild lung diseases. Boesch and Acton compared $\mathrm{FEV}_{1}$ slope of change 1 year pre- and post-ARS, and showed a significant improvement in pulmonary function only in the group with severe lung disease (i.e., $\left.\mathrm{FEV}_{1}<60 \%\right)^{19}$

\section{Nutrition Status}

Severe refractory GERD affects nutrition, stunts growth, and slows development. Controlling this conversely improves nutrition in $\mathrm{CF}$ patients, gives fewer CF exacerbations, and reduces the rate of $\mathrm{FEV}_{1}$ decline. Nutritional supplementation via gastrostomy is known to be beneficial due to increased calorific requirements of chronic disease. ${ }^{23}$ The majority of pediatric CF patients in this systematic review had a gastrostomy sited at the time of or prior to their fundoplication. However, gastrostomy placement may exacerbate gastroesophageal reflux. Hence, if it is undertaken in a patient with medically managed GERD, adequate investigation (preferably with pH-impedance monitoring) and consideration ought to be given to ARS. In Vinocur et al's study, all infants with CF who were failing to thrive prior to fundoplication had improved weight centiles following ARS with gastrostomy insertion. ${ }^{11}$ Sheikh et al also showed significant weight gain in their cohort of 48 patients with $\mathrm{CF}$ and GERD but not in BMI 2 years after fundoplication. ${ }^{20}$ The groups that exhibited weight gain were those with mild lung disease and those who received gastrostomy feeds. The majority of children in Boesch and Acton's article had concurrent gastrostomy insertion with ARS, but the only significant improved nutritional parameter was BMI in those with mild lung disease. ${ }^{19}$ Therefore, those most likely to attain nutritional benefits are infants and those with milder disease. This is probably related to supplemental feeding via gastrostomy and is evidence for early intervention to help preserve lung function. 


\section{Postoperative Complications of ARS}

Evidence suggests that the pediatric patient with CF tolerates fundoplication well. Despite being a high-risk group often requiring preoperative optimization, there were no deaths as a result of ARS. A recent systematic review of fundoplication in the children with and without neurological impairment showed poorly reported complication rates, ranging from 7 to $37 \% .{ }^{24}$ The present study ascertained that the ARS-related complication rate in pediatric CF patients was $18 \%$. To note, the earlier and smaller series did not report any complications. ${ }^{11,18}$ The latter two and larger series had a more comprehensive analysis of their surgical complications. ${ }^{19,20}$

\section{Follow-up Time}

Long-term follow-up data are largely missing in this cohort of pediatric patients with CF and GERD. The majority of cases were followed up for 2 years post-ARS. The evaluation of long-term outcomes in terms of symptoms, lung function, and complications of fundoplication would help assess the efficacy and morbidity of ARS in children with CF.

\section{Multidisciplinary Approach and Future Study Design}

Primary outcome measures in CF have altered dramatically in recent decades. With increasing life expectancy, there has been a shift in policy for lung preservation and prevention of long-term complications such as malignancy secondary to GERD. Therefore, ARS plays a crucial role in the management of these often silent aspiration events and patients must be carefully selected. Fundoplication is a prerequisite in $\mathrm{CF}$ patients prior to lung transplantation regardless of evidence due to the critical protection of any potential damage to the donor organ. Traditionally, pediatric patients have Nissen fundoplication performed with a complete wrap. The potential morbidity of wrap migration, failure, and gas bloat symptoms entertain the notion of jejunal feeding or partial fundoplication (e.g., anterior or posterior) to aid reflux reduction while minimizing adverse symptomatology. Jejunal feeding, in a child who would otherwise feed orally, should be the last resort or in those who pose a high risk to ARS. CF is a well-funded disease cohort in the United Kingdom with a multidisciplinary approach. Hence, it is becoming increasingly important for pediatric surgeons to be part of this team, not just for meconium ileus, distal intestinal obstruction syndrome, and venous access but also for optimal GERD management.

The primary aim of this study was to evaluate the incidence of GERD in CF and the outcomes of ARS. In order for the latter to be comprehensively concluded, future study designs must prospectively analyze this cohort, adapt an multidisciplinary approach, use standardized preoperative investigative methods (such as pH-impedance monitoring with symptom correlation scores), as well as measure unified outcome data (including the rate of decline in lung function, nutrition, and quality of life scores).

\section{General Limitations}

CF is a rare condition and life expectancy continues to increase. Between 1987 and 1991, the median predicted life expectancy was 29 years compared with the recent life expectancy of 44 years (2014-2018). ${ }^{25}$ The studies analyzed in this review included those dating back to 1982 and we recognize CF management has changed and advanced greatly since then with significant improvement in life expectancy. Limitations of the present systematic review include the collection of heterogeneous data or limited cohort size and nonuniform outcomes. Therefore, caution must be taken when drawing firm conclusions. Two studies included several patients over the age of 18 years; however, the vast majority of patients in these larger cohorts were in the pediatric age range and thus were included to add valuable data to our review. ${ }^{15,20}$

\section{Conclusion}

Approximately half the infants and children with CF have GERD, with around 1 in 10 of these refractory to management. While the evidence is limited, ARS has shown to slow the deterioration of lung function, and along with gastrostomy insertion, ARS can improve nutritional status in infants and children with CF. The surgeon should be part of the $\mathrm{CF}$ multidisciplinary team to investigate GERD and consider ARS. This can help save critical lung function and reduce cancer risk in a cohort with an ever-optimistic life expectancy.

Conflict of Interest

None declared.

\section{References}

1 Rosen R, Vandenplas Y, Singendonk M, et al. Pediatric Gastroesophageal Reflux Clinical Practice Guidelines: Joint Recommendations of the North American Society for Pediatric Gastroenterology, Hepatology, and Nutrition and the European Society for Pediatric Gastroenterology, Hepatology, and Nutrition. J Pediatr Gastroenterol Nutr 2018;66(03):516-554

2 Pauwels A, Decraene A, Blondeau K, et al. Bile acids in sputum and increased airway inflammation in patients with cystic fibrosis. Chest 2012;141(06):1568-1574

3 Navarro J, Rainisio M, Harms HK, et al. Factors associated with poor pulmonary function: cross-sectional analysis of data from the ERCF. European Epidemiologic Registry of Cystic Fibrosis. Eur Respir J 2001;18(02):298-305

4 Pencharz PB, Durie PR. Pathogenesis of malnutrition in cystic fibrosis, and its treatment. Clin Nutr 2000;19(06):387-394

5 Maqbool A, Pauwels A. Cystic fibrosis and gastroesophageal reflux disease. J Cyst Fibros 2017;16(Suppl 2):S2-S13

6 Mauritz FA, van Herwaarden-Lindeboom MY, Stomp W, et al. The effects and efficacy of antireflux surgery in children with gastroesophageal reflux disease: a systematic review. J Gastrointest Surg 2011;15(10):1872-1878

7 Baerg J, Thorpe D, Gasior A, Vannix R, Tagge E, St Peter S. Factors associated with mortality after Nissen fundoplication in children. Eur J Pediatr Surg 2015;25(03):277-283

8 Moher D, Liberati A, Tetzlaff J, Altman DGPRISMA Group. Preferred Reporting Items for Systematic Reviews and Meta-Analyses: the PRISMA statement. Int J Surg 2010;8(05):336-341

9 OCEBM Levels of Evidence Working Group. The Oxford 2011 Levels of Evidence. Oxford Centre for Evidence-Based Medicine. Available at: http://www.cebm.net/index.aspx?o=5653. Accessed August 15, 2020 
10 Slim K, Nini E, Forestier D, Kwiatkowski F, Panis Y, Chipponi J. Methodological index for non-randomized studies (MINORS): development and validation of a new instrument. ANZ J Surg 2003;73(09):712-716

11 Vinocur CD, Marmon L, Schidlow DV, Weintraub WH. Gastroesophageal reflux in the infant with cystic fibrosis. Am J Surg 1985;149(01):182-186

12 Gustafsson PM, Fransson SG, Kjellman NI, Tibbling L. Gastrooesophageal reflux and severity of pulmonary disease in cystic fibrosis. Scand J Gastroenterol 1991;26(05):449-456

13 Malfroot A, Dab I. New insights on gastro-oesophageal reflux in cystic fibrosis by longitudinal follow up. Arch Dis Child 1991;66 (11):1339-1345

14 Heine RG, Button BM, Olinsky A, Phelan PD, Catto-Smith AG. Gastro-oesophageal reflux in infants under 6 months with cystic fibrosis. Arch Dis Child 1998;78(01):44-48

15 BrodzickiJ, Trawińska-Bartnicka M, Korzon M. Frequency, consequences and pharmacological treatment of gastroesophageal reflux in children with cystic fibrosis. Med Sci Monit 2002;8(07):CR529-CR537

16 Dziekiewicz MA, Banaszkiewicz A, Urzykowska A, et al. Gastroesophageal reflux disease in children with cystic fibrosis. Adv Exp Med Biol 2015;873:1-7

17 Hauser B, De Schepper J, Malfroot A, et al. Gastric emptying and gastro-oesophageal reflux in children with cystic fibrosis. J Cyst Fibros 2016;15(04):540-547
18 Bendig DW, Seilheimer DK, Wagner ML, Ferry GD, Barrison GM. Complications of gastroesophageal reflux in patients with cystic fibrosis. J Pediatr 1982;100(04):536-540

19 Boesch RP, Acton JD. Outcomes of fundoplication in children with cystic fibrosis. J Pediatr Surg 2007;42(08):1341-1344

20 Sheikh SI, Ryan-Wenger NA, McCoy KS. Outcomes of surgical management of severe GERD in patients with cystic fibrosis. Pediatr Pulmonol 2013;48(06):556-562

21 Jolley SG, Johnson DG, Herbst JJ, Pena A, Garnier R. An assessment of gastroesophageal reflux in children by extended $\mathrm{pH}$ monitoring of the distal esophagus. Surgery 1978;84(01):16-24

22 Martigne L, Delaage PH, Thomas-Delecourt F, Bonnelye G, Barthélémy P, Gottrand F. Prevalence and management of gastroesophageal reflux disease in children and adolescents: a nationwide crosssectional observational study. Eur J Pediatr 2012;171(12):1767-1773

23 Walker SA, Gozal D. Pulmonary function correlates in the prediction of long-term weight gain in cystic fibrosis patients with gastrostomy tube feedings. J Pediatr Gastroenterol Nutr 1998;27(01):53-56

24 Cullis PS, Siminas S, Losty PD. Efficacy of antireflux surgery in children with or without neurological impairment: a systematic review. Br J Surg 2020;107(06):636-646

25 Cystic Fibrosis Foundation Patient Registry. 2018 Annual Data Report. 2019. Available at: https://www.cff.org/Research/ResearcherResources/Patient-Registry/2018-Patient-Registry-Annual-Data-Report.pdf Accessed August 15, 2020 